\title{
ON THE VOLUME OF THE ZERO CELL OF A CLASS OF ISOTROPIC POISSON HYPERPLANE TESSELLATIONS
}

\author{
JULIA HÖRRMANN * AND \\ DANIEL HUG, ${ }^{* * *}$ Karlsruhe Institute of Technology
}

\begin{abstract}
We study a parametric class of isotropic but not necessarily stationary Poisson hyperplane tessellations in $n$-dimensional Euclidean space. Our focus is on the volume of the zero cell, i.e. the cell containing the origin. As a main result, we obtain an explicit formula for the variance of the volume of the zero cell in arbitrary dimensions. From this formula we deduce the asymptotic behaviour of the volume of the zero cell as the dimension goes to $\infty$.
\end{abstract}

Keywords: Poisson hyperplane tessellation; Poisson-Voronoi tessellation; zero cell; typical cell; variance; high dimensions

2010 Mathematics Subject Classification: Primary 60D05

Secondary 52A22

\section{Introduction}

The majority of contributions to random tessellations is devoted to investigations in low and fixed dimensions. In particular, there exist only a few results on random tessellations in high dimensions, that is, with focus on asymptotic aspects as the dimension goes to $\infty$. Recently, the typical cell of a stationary Poisson-Voronoi tessellation in high dimensions has been studied in [1], [14], and [27]. Alishahi and Sharifitabar [1] investigated the asymptotic behaviour of the volume and the shape of the typical cell of a stationary Poisson-Voronoi tessellation as the dimension $n$ of the space goes to $\infty$. In particular, they showed that the variance of the volume of the typical cell converges to 0 exponentially fast as $n \rightarrow \infty$, whereas it is well known that the expected volume is independent of the dimension. In the course of their investigation, they made use of an explicit formula for the variance of the volume of the typical cell in arbitrary dimensions. The asymptotic behaviour of the volume of the typical cell was studied earlier in the more general context of the nearest-neighbour analysis by Newman et al. in [17] and [18]. In [18, Theorem 10], they showed that, if the intensity of the underlying Poisson process is $\gamma$ then the $k$ th moment of the volume of the typical cell converges to $\gamma^{-k}$ as the dimension goes to $\infty$ and, therefore, in particular, the volume of the typical cell converges in distribution to $\gamma^{-1}$.

In this work we consider a parametric class of Poisson hyperplane tessellations and focus on the volume of the cell containing the origin (the zero cell). It is then natural to explore whether an asymptotic behaviour similar to that of the typical cell of a stationary PoissonVoronoi tessellation is exhibited by the zero cell in the present more general class of random tessellations. An interesting family of not necessarily stationary or isotropic Poisson hyperplane

Received 6 December 2011; revision received 16 October 2013.

* Postal address: Department of Mathematics, Karlsruhe Institute of Technology, D-76128 Karlsruhe, Germany.

**Email address: daniel.hug@kit.edu 
tessellations is introduced in connection with the investigation of Kendall's conjecture in [8]. In the isotropic case, these Poisson hyperplane tessellations are completely determined by two parameters, the intensity $\gamma \in(0, \infty)$ and the distance exponent $r \in(0, \infty)$. For a special choice of the distance exponent and the intensity, the zero cell is equal in distribution to the typical cell of a stationary Poisson-Voronoi tessellation (cf. [4, Sections 5.2 and 5.3.2] and [8]). Therefore, this family of isotropic Poisson hyperplane tessellations provides a general framework for investigating tessellations in high dimensions. Trying to extend the approach of Alishahi and Sharifitabar [1] to the wider context of the zero cell of this family of Poisson hyperplane tessellations, we came across the need for a formula for the variance of the volume of the zero cell. Finding a manageable expression turned out to be a rather complex issue. In fact, the formulae presented here mark the starting point of a more detailed study of the asymptotic behaviour of characteristics of the zero cell as the dimension goes to $\infty$. Results concerning lower-dimensional sections of the zero cell and other shape characteristics as well as a connection to the hyperplane conjecture will be considered separately.

In the following, we give a more detailed overview of our results. A precise description of the particular (parametric) model of a Poisson hyperplane tessellation used here is given in Section 2. For this model, we then derive, in Section 3, an explicit expression for the expectation and bounds for the moments of the volume of the zero cell in Proposition 1. An explicit expression for the second moment and the variance of the volume of the zero cell is provided in Theorem 1. These results follow from a sequence of lemmas which make use of integral geometric transformations and the symmetries of the geometric situation. In Theorem 2 we deduce bounds for the variance of the volume of the zero cell which involve auxiliary quantities $D(n, r)$ and $E(n, r)$. These quantities have to be evaluated and bounded from above and below for a given distance exponent $r$ and an intensity $\gamma$. In Corollary 1 we consider the zero cell of a Poisson hyperplane tessellation with constant distance exponent $r$. The choice $r=1$ corresponds to a stationary Poisson hyperplane tessellation. For constant intensity, we then prove that all moments as well as the variance of the volume of the zero cell go to $\infty$ as the dimension $n$ goes to $\infty$.

In order to fix the expected volume of the zero cell, independent of the dimension, the intensity of the underlying Poisson hyperplane process can be chosen appropriately as a function of the dimension $n$. However, it follows from our bounds that, as long as the distance exponent $r$ is fixed, the variance of the volume of the zero cell still goes to $\infty$ as $n$ goes to $\infty$. The investigation in Section 4 thus suggests that in order to ensure that the variance converges to 0 , the distance exponent $r$ has to be adjusted to the dimension $n$. In Corollary 2 we summarize the case where the distance exponent $r$ is proportional to the dimension $n$, i.e. $r=a n$ with a fixed factor $a \in(0, \infty)$. For constant intensity, we show that the expectation and the moments of the volume of the zero cell now all converge to 0 as the dimension $n$ goes to $\infty$.

In Theorem 3 we finally consider the situation where the distance exponent $r$ is proportional to the dimension, i.e. $r=a n$ with some fixed $a>0$, and the intensity $\widehat{\gamma}(a, n)$ is chosen as a function of the dimension $n$ and the factor $a$ in such a way that the expected volume of the zero cell is equal to a positive constant. In this case we prove that the variance of the volume of the zero cell converges to 0 at an exponential speed of optimal order as $n \rightarrow \infty$. In particular, the volume of the zero cell converges in distribution. In the special case $r=n$ (i.e. $a=1$ ), we fully recover results for the typical cell of a Poisson-Voronoi tessellation obtained in [1]. The present more general approach applies to a larger class of tessellations and admits various other extensions and variations that will be discussed in detail in subsequent work. 


\section{Preliminaries}

In the following, we mainly use the notation and terminology of the monograph [23]. We work in an $n$-dimensional real Euclidean vector space $\mathbb{R}^{n}, n \geq 2$, with scalar product $\langle\cdot, \cdot\rangle$ and norm $\|\cdot\|$. The unit ball $\left\{x \in \mathbb{R}^{n}:\|x\| \leq 1\right\}$ centred at the origin $o$ is denoted by $B^{n}$, its boundary is the unit sphere $S^{n-1}$. For $k \in\{0, \ldots, n\}$, the Grassmannian of $k$-dimensional linear subspaces of $\mathbb{R}^{n}$ is denoted by $G(n, k)$, and the affine Grassmannian of $k$-dimensional affine subspaces ( $k$-flats) by $A(n, k)$; both are equipped with their standard topologies. For $u \in S^{n-1}$ and $t \in[0, \infty)$, we write

$$
H(u, t):=\left\{x \in \mathbb{R}^{n}:\langle x, u\rangle=t\right\}, \quad H^{-}(u, t):=\left\{x \in \mathbb{R}^{n}:\langle x, u\rangle \leq t\right\} .
$$

The Lebesgue measure on $\mathbb{R}^{n}$ is denoted by $\lambda$. For $E \in G(n, k)$, the Lebesgue measure on $E$ is denoted by $\lambda_{E}$. Besides, we define $S_{E}^{k-1}:=E \cap S^{n-1}$ and $H_{E}(u, t):=E \cap H(u, t)$ for $u \in S_{E}^{k-1}$ and $k \in\{1, \ldots, n\}$. The $s$-dimensional Hausdorff measure is denoted by $\mathscr{H}^{s}$, where $s \geq 0$. For $s=n$, we sometimes refer to it as the $n$-dimensional volume $V_{n}$. A frequently occurring constant is the volume of the unit ball,

$$
\kappa_{k}:=\lambda_{k}\left(B^{k}\right)=\frac{\pi^{k / 2}}{\Gamma(k / 2+1)}
$$

for $k \in \mathbb{N}_{0}$. The surface area of the unit sphere $S^{k-1}$ for $k \in \mathbb{N}$ is given by

$$
\omega_{k}:=\mathscr{H}^{k-1}\left(S^{k-1}\right)=k \kappa_{k}=\frac{2 \pi^{k / 2}}{\Gamma(k / 2)} .
$$

We repeatedly use the fact that

$$
\int_{0}^{\pi / 2}(\sin \varphi)^{\alpha}(\cos \varphi)^{\beta} \mathrm{d} \varphi=\frac{1}{2} \frac{\Gamma((\alpha+1) / 2) \Gamma((\beta+1) / 2)}{\Gamma((\alpha+\beta+2) / 2)}
$$

for $\alpha, \beta>-1$; see [2, Equation (5.6)] or [26, Equation (12.42)]. In the following, we often use the connection between the beta and the gamma functions (see [26, Equation (12.41)]) and the approximation of the gamma function given by

$$
\Gamma(x)=\sqrt{2 \pi} x^{x-1 / 2} \exp \left(-x+\frac{\theta(x)}{12 x}\right), \quad x>0,
$$

where $\theta(x) \in(0,1)$ (see [26, Equation (12.33)] or [2, p. 24]). For $m \in \mathbb{N}$ and $x_{1}, \ldots, x_{m} \in \mathbb{R}^{n}$, we denote by $\left[x_{1}, \ldots, x_{m}\right]$ the convex hull and by $\operatorname{span}\left\{x_{1}, \ldots, x_{m}\right\}$ the linear hull of $x_{1}, \ldots, x_{m}$.

The family of nonempty, compact, convex subsets of $\mathbb{R}^{n}$ is denoted by $\mathcal{K}^{n}$. For a topological space $(T, \mathcal{T})$, a measure is always defined on the $\sigma$-algebra $\mathscr{B}(T)$ of Borel sets of $T$, i.e. the smallest $\sigma$-algebra containing the open sets $\mathcal{T}$. We write $\mu^{k}:=\mu \otimes \cdots \otimes \mu$, with $k$ factors $\mu$, for the $k$-fold product of a measure $\mu$. $\mathrm{By} \mathrm{SO}_{n}$ we denote the group of proper (i.e. rotation preserving) rotations on $\mathbb{R}^{n}$, and $v_{n}$ is the unique Haar probability measure on $\mathrm{SO}_{n}$.

The following setting has previously been considered in a more general, not necessarily isotropic, framework in the context of Kendall's problem [8] (see also [4] and [6]). Let $(\Omega, \mathcal{A}, \mathbb{P})$ denote the underlying probability space. Furthermore, let $X$ be a Poisson hyperplane process in $\mathbb{R}^{n}$, i.e. a Poisson point process in the space $A(n, n-1)$. Subsequently, we identify a simple counting measure with its support, so that, for a Borel set $A \subset A(n, n-1)$, both the notation 
$X(A)$ and $\operatorname{card}(X \cap A)$ denote the number of elements of $X$ in A. We assume that the intensity measure $\Theta(\cdot)=\mathbb{E} X(\cdot)$ of $X$ is of the form

$$
\Theta(\cdot)=\frac{2 \gamma}{n \kappa_{n}} \int_{S^{n-1}} \int_{0}^{\infty} \mathbf{1}\{H(u, t) \in \cdot\} t^{r-1} \mathrm{~d} t \mathscr{H}^{n-1}(\mathrm{~d} u)
$$

with $\gamma>0$ and $r \in(0, \infty)$. We refer to $\gamma$ as the intensity and to $r$ as the distance exponent of the hyperplane process $X$. Clearly, $\Theta$ is rotation invariant for all $r>0$. Furthermore, $\Theta$ is translation invariant only for $r=1$. Observe that a calculation similar to that required for (5) below implies that $\Theta(\{H \in A(n, n-1): H \cap[-e, e] \neq \varnothing\})=\Theta(\{H \in A(n, n-1): H \cap$ $[0,2 e] \neq \varnothing\}$ ) for $e \in S^{n-1}$ if and only if $r=1$. (It is well known that the intensity measure is translation invariant for $r=1$; cf. [23, Equation (4.33)] and the reflection symmetry of $\mathcal{H}^{n-1}$ on the unit sphere.) Therefore, since $X$ is a Poisson process, $X$ is always isotropic but stationary only for $r=1$.

The random polytope

$$
Z_{0}:=\bigcap_{H \in X} H^{-}
$$

is the zero cell of the hyperplane process $X$, where $H^{-}$denotes the (almost surely uniquely determined) closed half-space bounded by $H$ which contains the origin. Clearly, $Z_{0}$ depends on $\gamma$ and $r$; although this dependence is not made explicit by our notation.

For the distance exponent $r=n$, the zero cell $Z_{0}$ is equal in distribution to the typical cell of a stationary Poisson-Voronoi tessellation (see [8]). More detailed information on the topic of random tessellations is provided in, e.g. [19], [23], [24, Chapter 6], and [25]. Poisson-Voronoi tessellations have been studied extensively in the literature; see, e.g. [7], [13], and [15]. Stationary Poisson hyperplane tessellations have been considered in, e.g. [9], [10], [11], and [12]. Recently, nonstationary Poisson hyperplane tessellations have also attracted some attention (cf. [8] and [21]). A review of recent results on random polytopes is given in [3], [20], [22], [23, Chapter 8], and [24, Chapter 7].

\section{A general formula for the variance}

Let $X$ be a Poisson hyperplane process in $\mathbb{R}^{n}$ with an intensity measure of the form (3). We assume that $\gamma>0$ and $r \in(0, \infty)$. By Fubini's theorem and basic properties of a Poisson process, we have

$$
\begin{aligned}
& \mathbb{E}\left[V_{n}\left(Z_{0}\right)^{k}\right]=\mathbb{E}\left[\int_{\mathbb{R}^{n}} \mathbf{1}_{Z_{0}}\left(x_{1}\right) \mathrm{d} x_{1} \cdots \int_{\mathbb{R}^{n}} \mathbf{1}_{Z_{0}}\left(x_{k}\right) \mathrm{d} x_{k}\right] \\
&=\int_{\left(\mathbb{R}^{n}\right)^{k}} \mathbb{P}\left(x_{1}, \ldots, x_{k} \in Z_{0}\right) \mathrm{d} x_{1} \cdots \mathrm{d} x_{k} \\
&=\int_{\left(\mathbb{R}^{n}\right)^{k}} \exp \left[-\frac{2 \gamma}{n \kappa_{n}} \int_{S^{n-1}} \int_{0}^{\infty} \mathbf{1}\left\{H(u, t) \cap\left[o, x_{1}, \ldots, x_{k}\right] \neq \varnothing\right\}\right. \\
&\left.\times t^{r-1} \mathrm{~d} t \mathcal{H}^{n-1}(\mathrm{~d} u)\right] \mathrm{d} x_{1} \cdots \mathrm{d} x_{k} .
\end{aligned}
$$

From (4) we now deduce lower and upper bounds for the moments of $V_{n}\left(Z_{0}\right)$. For $e \in S^{n-1}$, we define

$$
c(n, r):=\int_{S^{n-1}}\langle e, u\rangle_{+}^{r} \mathscr{H}^{n-1}(\mathrm{~d} u)=\pi^{(n-1) / 2} \frac{\Gamma((r+1) / 2)}{\Gamma((r+n) / 2)},
$$


which is indeed independent of the choice of the unit vector $e$. The explicit value is determined by a suitable decomposition of the spherical Lebesgue measure (see [16, Equation (1.41)]) and by an application of (1).

The following result provides bounds from above and below for the moments of the volume of the zero cell. Note that the ratio of the upper and the lower bound is given by the ratio of the corresponding values of the gamma functions in these bounds.

Proposition 1. For $k \in \mathbb{N}$, we have

$$
\Gamma\left(\frac{n}{r}+1\right)^{k} \kappa_{n}^{k}\left(\frac{n \kappa_{n} r}{2 \gamma c(n, r)}\right)^{k n / r} \leq \mathbb{E}\left[V_{n}\left(Z_{0}\right)^{k}\right] \leq \Gamma\left(\frac{k n}{r}+1\right) \kappa_{n}^{k}\left(\frac{n \kappa_{n} r}{2 \gamma c(n, r)}\right)^{k n / r} .
$$

In particular, for $k=1$, we obtain

$$
\mathbb{E}\left[V_{n}\left(Z_{0}\right)\right]=\Gamma\left(\frac{n}{r}+1\right) \kappa_{n}\left(\frac{n \kappa_{n} r}{2 \gamma c(n, r)}\right)^{n / r} .
$$

Proof. Starting with (4), introducing polar coordinates, and by symmetry, we obtain

$$
\begin{array}{r}
\mathbb{E}\left[V_{n}\left(Z_{0}\right)^{k}\right] \\
=k \int_{0}^{\infty} \int_{0}^{s_{1}} \cdots \int_{0}^{s_{1}} \int_{\left(S^{n-1}\right)^{k}} \exp \left[-\frac{2 \gamma}{n \kappa_{n}}\right. \\
\times \int_{S^{n-1}} \int_{0}^{\infty} \mathbf{1}\left\{H(u, t) \cap\left[o, s_{1} v_{1}, \ldots, s_{k} v_{k}\right] \neq \varnothing\right\} \\
\left.\times t^{r-1} \mathrm{~d} t \mathscr{H}^{n-1}(\mathrm{~d} u)\right] s_{1}^{n-1} \cdots s_{k}^{n-1}
\end{array}
$$

Since

$$
\begin{aligned}
\int_{S^{n-1}} & \int_{0}^{\infty} \mathbf{1}\left\{H(u, t) \cap\left[o, s_{1} v_{1}\right] \neq \varnothing\right\} t^{r-1} \mathrm{~d} t \mathscr{H}^{n-1}(\mathrm{~d} u) \\
& =\int_{S^{n-1}} \int_{0}^{\infty} \mathbf{1}\left\{0 \leq t \leq\left\langle s_{1} v_{1}, u\right\rangle_{+}\right\} t^{r-1} \mathrm{~d} t \mathscr{H}^{n-1}(\mathrm{~d} u) \\
& =\frac{1}{r} s_{1}^{r} c(n, r),
\end{aligned}
$$

the upper bound follows easily.

For the lower bound, we observe that $H(u, t) \cap\left[o, x_{1}, \ldots, x_{k}\right] \neq \varnothing$ if and only if there is some $i \in\{1, \ldots, k\}$ such that $H(u, t) \cap\left[o, x_{i}\right] \neq \varnothing$. Hence, $\mathbb{E}\left[V_{n}\left(Z_{0}\right)^{k}\right]$ can be bounded from 
below by

$$
\begin{aligned}
\int_{\left(\mathbb{R}^{n}\right)^{k}} & \exp \left[-\frac{2 \gamma}{n \kappa_{n}} \int_{S^{n-1}} \int_{0}^{\infty} \sum_{i=1}^{k} \mathbf{1}\left\{H(u, t) \cap\left[o, x_{i}\right] \neq \varnothing\right\} t^{r-1} \mathrm{~d} t \mathcal{H}^{n-1}(\mathrm{~d} u)\right] \mathrm{d} x_{1} \cdots \mathrm{d} x_{k} \\
= & \left(\int_{\mathbb{R}^{n}} \exp \left[-\frac{2 \gamma\left\|x_{1}\right\|^{r}}{n \kappa_{n} r} c(n, r)\right] \mathrm{d} x_{1}\right)^{k} .
\end{aligned}
$$

Now the assertion follows by a straightforward calculation.

Remark 1. The lower bound in Proposition 1 can also be obtained by an application of Hölder's inequality. Moreover, for fixed $r$ and $k$, the ratio of the upper and the lower bound is of the order $n^{(1-k) / 2} k^{k n / r}$.

For the statement of Theorem 1, which provides formulae for the second moment and the variance of $V_{n}\left(Z_{0}\right)$, we need the constant

$$
b_{n, 2}:=\frac{\omega_{n-1} \omega_{n}}{4 \pi}
$$

and the auxiliary functions

$$
\alpha(t, \varphi):=\arctan \left(\frac{t-\cos \varphi}{\sin \varphi}\right) \in\left(-\frac{\pi}{2}, \frac{\pi}{2}\right)
$$

and

$$
F_{r}(t, \varphi):=\frac{1}{c(2, r)}\left(t^{r} \int_{-\pi / 2}^{\alpha(t, \varphi)}(\cos \theta)^{r} \mathrm{~d} \theta+\int_{\alpha(t, \varphi)-\varphi}^{\pi / 2}(\cos \theta)^{r} \mathrm{~d} \theta\right)
$$

for $(t, \varphi) \in[0,1] \times(0, \pi)$. In the following, we will use the fact that

$$
\frac{1}{2} \leq F_{r}(t, \varphi) \leq t^{r}+1 \leq 2
$$

for all $(t, \varphi) \in[0,1] \times(0, \pi)$, which follows from Remark 3(a) and (b) below.

Theorem 1. Let $X$ be a Poisson hyperplane process in $\mathbb{R}^{n}$ with an intensity measure of the form (3) with intensity $\gamma>0$ and distance exponent $r \in(0, \infty)$. Then

$$
\mathbb{E}\left[V_{n}\left(Z_{0}\right)^{2}\right]=\frac{8 \pi b_{n, 2}}{r} \Gamma\left(\frac{2 n}{r}\right)\left(\frac{n \kappa_{n} r}{2 \gamma c(n, r)}\right)^{2 n / r} \int_{0}^{\pi} \int_{0}^{1} \frac{t^{n-1}}{F_{r}(t, \varphi)^{2 n / r}}(\sin \varphi)^{n-2} \mathrm{~d} t \mathrm{~d} \varphi
$$

and

$$
\begin{aligned}
\operatorname{var}\left[V_{n}\left(Z_{0}\right)\right]= & \frac{8 \pi b_{n, 2}}{r} \Gamma\left(\frac{2 n}{r}\right)\left(\frac{n \kappa_{n} r}{2 \gamma c(n, r)}\right)^{2 n / r} \\
& \times \int_{0}^{\pi} \int_{0}^{1}\left(\frac{1}{F_{r}(t, \varphi)^{2 n / r}}-\frac{1}{\left(t^{r}+1\right)^{2 n / r}}\right) t^{n-1}(\sin \varphi)^{n-2} \mathrm{~d} t \mathrm{~d} \varphi .
\end{aligned}
$$

Proof. The formula for $\mathbb{E}\left[V_{n}\left(Z_{0}\right)^{2}\right]$ is implied by Lemma 3 and Lemma 4 below, and straightforward calculations. The formula for $\operatorname{var}\left[V_{n}\left(Z_{0}\right)\right]$ then follows if we also use the representation of $\left(\mathbb{E}\left[V_{n}\left(Z_{0}\right)\right]\right)^{2}$ as a double integral, i.e.

$$
\left(\mathbb{E}\left[V_{n}\left(Z_{0}\right)\right]\right)^{2}=\frac{8 \pi b_{n, 2}}{r} \Gamma\left(\frac{2 n}{r}\right)\left(\frac{n \kappa_{n} r}{2 \gamma c(n, r)}\right)^{2 n / r} \int_{0}^{\pi} \int_{0}^{1} \frac{t^{n-1}}{\left(t^{r}+1\right)^{2 n / r}}(\sin \varphi)^{n-2} \mathrm{~d} t \mathrm{~d} \varphi,
$$


which is a consequence of the special case $k=1$ of Proposition 1 and of the fact that

$$
\int_{0}^{1} \frac{t^{n-1}}{\left(t^{r}+1\right)^{2 n / r}} \mathrm{~d} t=\frac{1}{2 r} \frac{\Gamma(n / r)^{2}}{\Gamma(2 n / r)},
$$

which follows from the substitution $z=\left(t^{r}+1\right)^{-1}$, the symmetry of the resulting integrand with respect to $\frac{1}{2}$, and basic calculations involving beta and gamma functions. Furthermore, we use the fact that

$$
n^{2} \kappa_{n}^{2}=4 \pi b_{n, 2} \int_{0}^{\pi}(\sin \varphi)^{n-2} \mathrm{~d} \varphi,
$$

which follows from the definition of $\kappa_{n}$ and $b_{n, 2}$, and from a special case of (1).

Remark 2. In Figure 1 and Figure 2 we present the numerical results for the variance based on Theorem 1. In small dimensions $(n=2,3,4)$ the numerical calculations of the variance for varying $r$ are plotted for $\gamma=1$. In this case observe that, by Proposition 1 and (2), we have $\lim _{r \rightarrow \infty} \mathbb{E}\left[V_{n}\left(Z_{0}\right)^{k}\right]=\kappa_{n}^{k}$ for $k \in \mathbb{N}$ and arbitrarily fixed $n \geq 2$. In addition, we study the
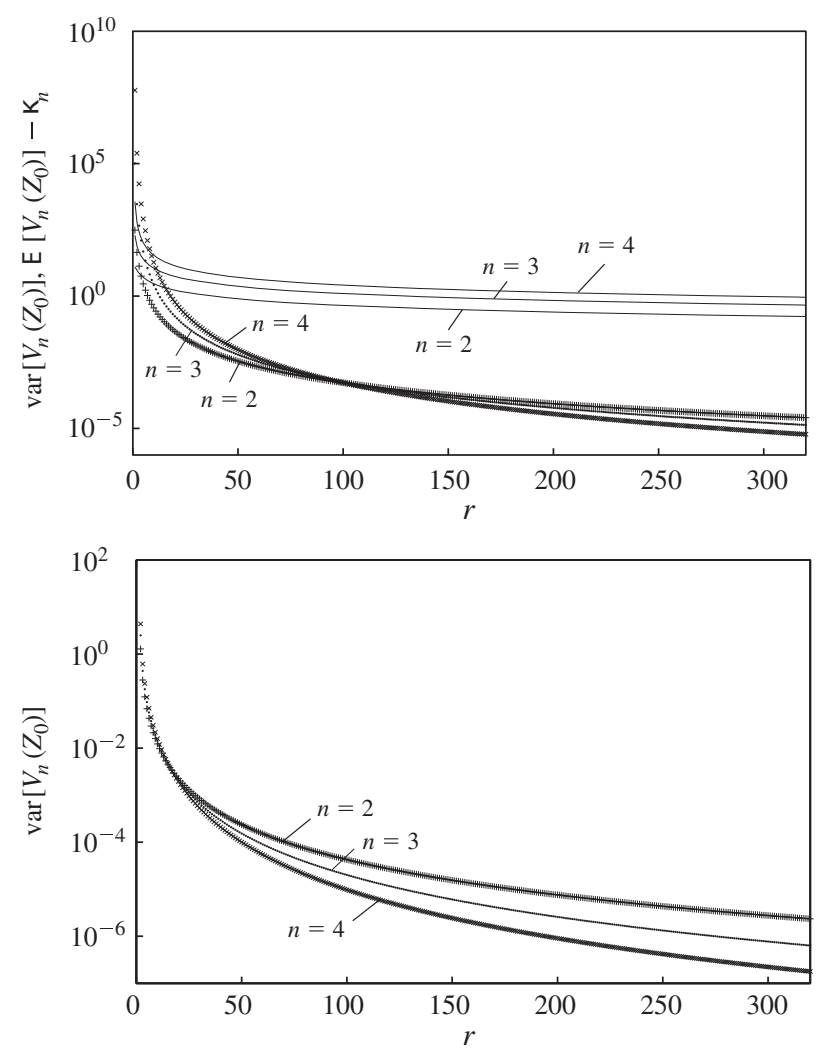

Figure 1: Numerical evaluation of the formula for the variance from Theorem 1 using the numerical integration functions of MATHEMATICA ${ }^{\circledR}$. Top: we fixed $\gamma=1$ and plotted $\mathbb{E}\left[V_{n}\left(Z_{0}\right)\right]-$ $\lim _{r \rightarrow \infty} \mathbb{E}\left[V_{n}\left(Z_{0}\right)\right]=\mathbb{E}\left[V_{n}\left(Z_{0}\right)\right]-\kappa_{n}$ as a solid line for comparison. Bottom: $\gamma$ was chosen in such a way that $\mathbb{E}\left[V_{n}\left(Z_{0}\right)\right]=1$. 

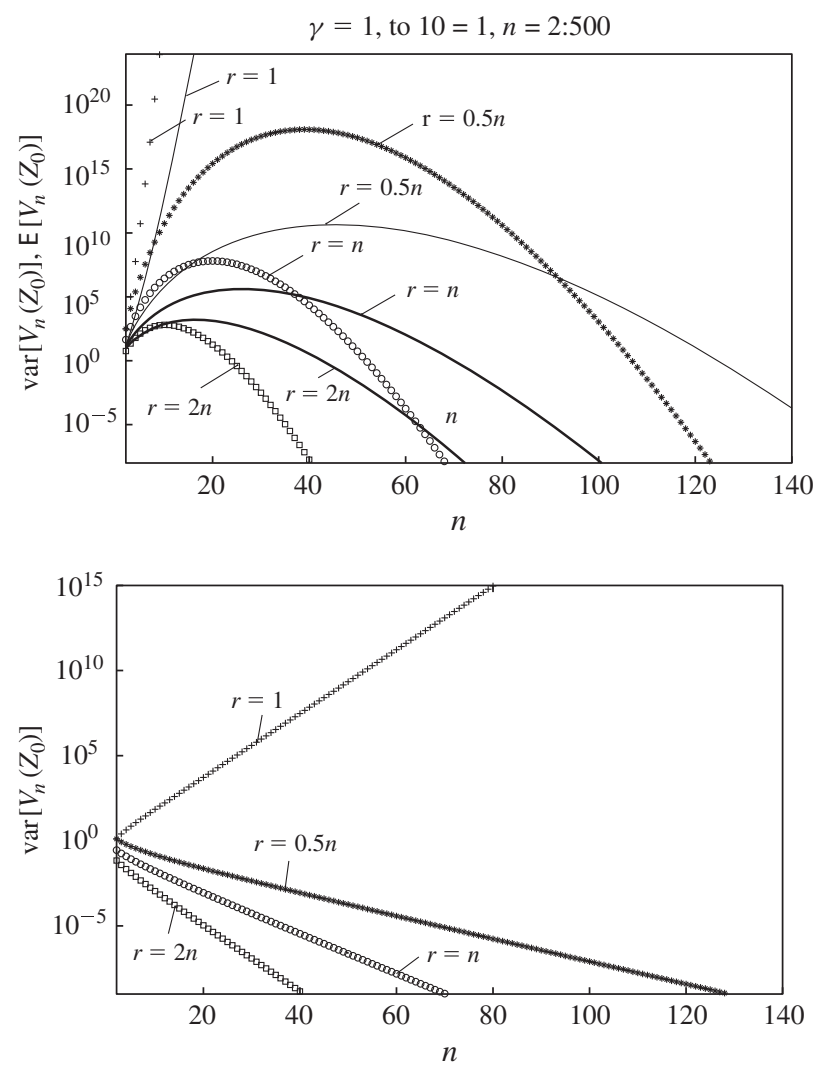

FIgURE 2: Numerical evaluation of the formula for the variance from Theorem 1 using the numerical integration functions of MATHEMATICA. Top: we fixed $\gamma=1$ and plotted $\mathbb{E}\left[V_{n}\left(Z_{0}\right)\right]$ as a solid line for comparison. Bottom: $\gamma$ was chosen in such a way that $\mathbb{E}\left[V_{n}\left(Z_{0}\right)\right]=1$.

choice

$$
\gamma=\frac{n \kappa_{n} r}{2 c(n, r)}\left(\Gamma\left(\frac{n}{r}+1\right) \kappa_{n}\right)^{r / n},
$$

which implies that $\mathbb{E}\left[V_{n}\left(Z_{0}\right)\right]=1$ and $\lim _{r \rightarrow \infty} \mathbb{E}\left[V_{n}\left(Z_{0}\right)^{k}\right]=1$ for $k \in \mathbb{N}$ and arbitrarily fixed $n \geq 2$ by Proposition 1 . Theorem 2 and Lemma 5 below, which will be proved in the following section, and (2) show that, for both choices of $\gamma$, we obtain

$$
\operatorname{var}\left[V_{n}\left(Z_{0}\right)\right] \leq C r^{-(n+1) / 2},
$$

where $C>0$ is a constant depending on $\gamma$ and $n$ in the first case and only on $n$ in the second. On the other hand, for specific choices of $r(r=1, r=0.5 n, r=n, r=2 n)$, the numerically determined values of the variance for varying dimension $n$ are plotted for $\gamma=1$ and $\gamma=n \kappa_{n} r\left(\Gamma(n / r+1) \kappa_{n}\right)^{r / n} /(2 c(n, r))$. The high-dimensional limiting behaviour of the moments and the variance is studied in the following section.

In the following remark we collect some facts that are helpful for a proper understanding of the formulae for the second moment and the variance of the volume of the zero cell in Theorem 1, which are used several times subsequently. 
Remark 3. (a) Let $\varphi \in(0, \pi)$ and $t \in[0,1]$. Then

$$
-\frac{\pi}{2}<\varphi-\frac{\pi}{2}=\alpha(0, \varphi) \leq \alpha(t, \varphi) \leq \alpha(1, \varphi)=\frac{\varphi}{2}<\frac{\pi}{2},
$$

since $t \mapsto \alpha(t, \varphi)$ is increasing on $[0,1]$.

(b) A special case of (1) is

$$
\int_{-\pi / 2}^{\pi / 2}(\cos \theta)^{r} \mathrm{~d} \theta=c(2, r) .
$$

The following lemmas lead successively to the explicit formulae for the second moment and the variance of the volume of the zero cell stated in Theorem 1. Lemma 1 and Lemma 2 are needed to prove Lemma 3, whereas Lemma 3 and Lemma 4 have been used directly in the proof of Theorem 1. In a first step, the integral representation of the second moment of $V_{n}\left(Z_{0}\right)$ from (4) will be simplified considerably by an application of a Blaschke-Petkantschin formula (cf. [23]). For $x_{1}, x_{2} \in \mathbb{R}^{n}$, let $\nabla_{2}\left(x_{1}, x_{2}\right)$ denote the area of the parallelogram spanned by these vectors. Furthermore, denote by $v_{2}^{n}$ the unique rotation invariant probability measure on $G(n, 2)$. As usual, $\left\{e_{1}, \ldots, e_{n}\right\}$ denotes the standard basis of $\mathbb{R}^{n}$.

Lemma 1. We have

$$
\begin{aligned}
\mathbb{E}\left[V_{n}\left(Z_{0}\right)^{2}\right]=b_{n, 2} \int_{\text {span }\left\{e_{1}, e_{2}\right\}^{2}} \exp \left[-\frac{2 \gamma}{n \kappa_{n}} \int_{S^{n-1}} \int_{0}^{\infty}\right. & \mathbf{1}\left\{H(u, t) \cap\left[o, x_{1}, x_{2}\right] \neq \varnothing\right\} t^{r-1} \\
& \left.\times \mathrm{d} t \mathscr{H}^{n-1}(\mathrm{~d} u)\right] \\
& \times \nabla_{2}\left(x_{1}, x_{2}\right)^{n-2} \lambda_{\operatorname{span}\left\{e_{1}, e_{2}\right\}}^{2}\left(\mathrm{~d}\left(x_{1}, x_{2}\right)\right) .
\end{aligned}
$$

Proof. From (4), the linear Blaschke-Petkantschin formula [23, Theorem 7.2.1], the rotation invariance of the spherical Lebesgue measure, and the invariance of $\nabla_{2}(\cdot, \cdot)$, we obtain

$$
\begin{aligned}
& \mathbb{E}\left[V_{n}\left(Z_{0}\right)^{2}\right] \\
& =b_{n, 2} \int_{G(n, 2)} \int_{L^{2}} \exp \left[-\frac{2 \gamma}{n \kappa_{n}} \int_{S^{n-1}} \int_{0}^{\infty} \mathbf{1}\left\{H(u, t) \cap\left[o, x_{1}, x_{2}\right] \neq \varnothing\right\} t^{r-1} \mathrm{~d} t \mathscr{H}^{n-1}(\mathrm{~d} u)\right] \\
& \times \nabla_{2}\left(x_{1}, x_{2}\right)^{n-2} \lambda_{L}^{2}\left(\mathrm{~d}\left(x_{1}, x_{2}\right)\right) v_{2}^{n}(\mathrm{~d} L) \\
& =b_{n, 2} \int_{\mathrm{SO}_{n}} \int_{\operatorname{span}\left\{e_{1}, e_{2}\right\}^{2}} \exp \left[-\frac{2 \gamma}{n \kappa_{n}} \int_{S^{n-1}} \int_{0}^{\infty} \mathbf{1}\left\{H(u, t) \cap\left[o, \vartheta x_{1}, \vartheta x_{2}\right] \neq \varnothing\right\}\right. \\
& \left.\times t^{r-1} \mathrm{~d} t \mathscr{H}^{n-1}(\mathrm{~d} u)\right] \nabla_{2}\left(\vartheta x_{1}, \vartheta x_{2}\right)^{n-2} \\
& \times \lambda_{\text {span }\left\{e_{1}, e_{2}\right\}}^{2}\left(\mathrm{~d}\left(x_{1}, x_{2}\right)\right) v_{n}(\mathrm{~d} \vartheta) \\
& =b_{n, 2} \int_{\mathrm{SO}_{n}} \int_{\operatorname{span}\left\{e_{1}, e_{2}\right\}^{2}} \exp \left[-\frac{2 \gamma}{n \kappa_{n}} \int_{S^{n-1}} \int_{0}^{\infty} \mathbf{1}\left\{H(\vartheta u, t) \cap\left[o, \vartheta x_{1}, \vartheta x_{2}\right] \neq \varnothing\right\}\right. \\
& \left.\times t^{r-1} \mathrm{~d} t \mathscr{H}^{n-1}(\mathrm{~d} u)\right] \nabla_{2}\left(x_{1}, x_{2}\right)^{n-2} \\
& \times \lambda_{\text {span }\left\{e_{1}, e_{2}\right\}}^{2}\left(\mathrm{~d}\left(x_{1}, x_{2}\right)\right) v_{n}(\mathrm{~d} \vartheta)
\end{aligned}
$$




$$
\begin{aligned}
=b_{n, 2} \int_{\text {span }\left\{e_{1}, e_{2}\right\}^{2}} \exp \left[-\frac{2 \gamma}{n \kappa_{n}} \int_{S^{n-1}} \int_{0}^{\infty}\right. & \mathbf{1}\left\{H(u, t) \cap\left[o, x_{1}, x_{2}\right] \neq \varnothing\right\} \\
& \left.\times t^{r-1} \mathrm{~d} t \mathscr{H}^{n-1}(\mathrm{~d} u)\right] \nabla_{2}\left(x_{1}, x_{2}\right)^{n-2} \\
& \times \lambda_{\operatorname{span}\left\{e_{1}, e_{2}\right\}}^{2}\left(\mathrm{~d}\left(x_{1}, x_{2}\right)\right),
\end{aligned}
$$

which yields the assertion of the lemma.

Next we simplify the inner double integral of the expression that was derived in Lemma 1 for $\mathbb{E}\left[V_{n}\left(Z_{0}\right)^{2}\right]$ by exploiting further the symmetry of the situation.

Lemma 2. For $x_{1}, x_{2} \in \operatorname{span}\left\{e_{1}, e_{2}\right\} \subset \mathbb{R}^{n}$, we have

$$
\begin{aligned}
\int_{S^{n-1}} & \int_{0}^{\infty} \mathbf{1}\left\{H(u, t) \cap\left[o, x_{1}, x_{2}\right] \neq \varnothing\right\} t^{r-1} \mathrm{~d} t \mathscr{H}^{n-1}(\mathrm{~d} u) \\
= & \frac{c(n, r)}{c(2, r)} \int_{0}^{2 \pi} \int_{0}^{\infty} \mathbf{1}\left\{H\left(\left(\begin{array}{c}
\cos \theta \\
\sin \theta
\end{array}\right), t\right) \cap\left[o, x_{1}, x_{2}\right] \neq \varnothing\right\} t^{r-1} \mathrm{~d} t \mathrm{~d} \theta .
\end{aligned}
$$

Proof. For $n=2$, there is nothing to prove. Hence, we can assume that $n \geq 3$. Let $E:=\operatorname{span}\left\{e_{1}, e_{2}\right\}$. The map

$$
F:\left\{\begin{aligned}
S_{E}^{1} \times\left(0, \frac{\pi}{2}\right) \times S_{E^{\perp}}^{n-3} & \rightarrow S^{n-1}, \\
\left(u_{1}, \theta, u_{2}\right) & \mapsto \cos (\theta) u_{1}+\sin (\theta) u_{2},
\end{aligned}\right.
$$

is injective and its image covers $S^{n-1}$ up to a set of measure 0. Its Jacobian is

$$
J F\left(u_{1}, \theta, u_{2}\right)=\cos (\theta)(\sin (\theta))^{n-3},
$$

and, hence, the area-coarea formula (cf. [5, Theorem 3.2.22]) yields

$$
\begin{aligned}
\int_{S^{n-1}} & \int_{0}^{\infty} \mathbf{1}\left\{H(u, t) \cap\left[o, x_{1}, x_{2}\right] \neq \varnothing\right\} t^{r-1} \mathrm{~d} t \mathscr{H}^{n-1}(\mathrm{~d} u) \\
= & \int_{S_{E}^{1}} \int_{0}^{\pi / 2} \int_{S_{E^{\perp}}^{n-3}} \int_{0}^{\infty} \mathbf{1}\left\{H\left(\cos (\theta) u_{1}+\sin (\theta) u_{2}, t\right) \cap\left[o, x_{1}, x_{2}\right] \neq \varnothing\right\} \\
& \times \cos (\theta)(\sin \theta)^{n-3} t^{r-1} \mathrm{~d} t \mathscr{H}^{n-3}\left(\mathrm{~d} u_{2}\right) \mathrm{d} \theta \mathscr{H}^{1}\left(\mathrm{~d} u_{1}\right) .
\end{aligned}
$$

Since $\left[o, x_{1}, x_{2}\right] \subset E$ and

$$
H\left(\cos (\theta) u_{1}+\sin (\theta) u_{2}, t\right) \cap E=H_{E}\left(u_{1}, \frac{t}{\cos \theta}\right)
$$

for $\theta \in[0, \pi / 2)$, we obtain

$$
\begin{aligned}
\int_{S^{n-1}} \int_{0}^{\infty} \mathbf{1}\left\{H(u, t) \cap\left[o, x_{1}, x_{2}\right] \neq \varnothing\right\} t^{r-1} \mathrm{~d} t \mathscr{H}^{n-1}(\mathrm{~d} u) \\
=(n-2) \kappa_{n-2} \int_{S_{E}^{1}} \int_{0}^{\pi / 2} \int_{0}^{\infty} \mathbf{1}\left\{H_{E}\left(u_{1}, \frac{t}{\cos \theta}\right) \cap\left[o, x_{1}, x_{2}\right] \neq \varnothing\right\} \\
\times \cos (\theta)(\sin \theta)^{n-3} t^{r-1} \mathrm{~d} t \mathrm{~d} \theta \mathscr{H}^{1}\left(\mathrm{~d} u_{1}\right) \\
=(n-2) \kappa_{n-2} \int_{S_{E}^{1}} \int_{0}^{\pi / 2} \int_{0}^{\infty} \mathbf{1}\left\{H_{E}\left(u_{1}, t\right) \cap\left[o, x_{1}, x_{2}\right] \neq \varnothing\right\} \\
\times(\cos \theta)^{r+1}(\sin \theta)^{n-3} t^{r-1} \mathrm{~d} t \mathrm{~d} \theta \mathscr{H}^{1}\left(\mathrm{~d} u_{1}\right)
\end{aligned}
$$




$$
\begin{aligned}
= & (n-2) \kappa_{n-2} \int_{0}^{\pi / 2}(\cos \theta)^{r+1}(\sin \theta)^{n-3} \mathrm{~d} \theta \\
& \times \int_{S^{1}} \int_{0}^{\infty} \mathbf{1}\left\{H\left(u_{1}, t\right) \cap\left[o, x_{1}, x_{2}\right] \neq \varnothing\right\} t^{r-1} \mathrm{~d} t \mathscr{H}^{1}\left(\mathrm{~d} u_{1}\right) \\
= & \frac{c(n, r)}{c(2, r)} \int_{0}^{2 \pi} \int_{0}^{\infty} \mathbf{1}\left\{H\left(\left(\begin{array}{c}
\cos \theta \\
\sin \theta
\end{array}\right), t\right) \cap\left[o, x_{1}, x_{2}\right] \neq \varnothing\right\} t^{r-1} \mathrm{~d} t \mathrm{~d} \theta,
\end{aligned}
$$

which completes the proof of the lemma.

Having simplified the inner integral of the expression found in Lemma 1 for $\mathbb{E}\left[V_{n}\left(Z_{0}\right)^{2}\right]$, we reduce the outer integral in the next lemma by again taking advantage of the problem's symmetry.

Lemma 3. We have

$$
\begin{aligned}
& \mathbb{E}\left[V_{n}\left(Z_{0}\right)^{2}\right]=8 \pi b_{n, 2} \int_{0}^{\pi} \int_{0}^{\infty} \int_{0}^{s} \exp \left[-\frac{2 \gamma}{n \kappa_{n}} \frac{c(n, r)}{c(2, r)}\right. \\
& \times \int_{0}^{2 \pi} \int_{0}^{\infty} \mathbf{1}\left\{H\left(\left(\begin{array}{c}
\cos \theta \\
\sin \theta
\end{array}\right), t\right) \cap\left[o, s\left(\begin{array}{c}
\cos \varphi \\
\sin \varphi
\end{array}\right),\left(\begin{array}{c}
u \\
0
\end{array}\right)\right] \neq \varnothing\right\} \\
&\left.\times t^{r-1} d t \mathrm{~d} \theta\right] s^{n-1} u^{n-1}(\sin \varphi)^{n-2} \mathrm{~d} u \mathrm{~d} s \mathrm{~d} \varphi .
\end{aligned}
$$

Proof. Combining Lemma 1 and Lemma 2, and introducing polar coordinates, we obtain $\mathbb{E}\left[V_{n}\left(Z_{0}\right)^{2}\right]$

$$
\begin{aligned}
&=b_{n, 2} \int_{0}^{2 \pi} \int_{0}^{2 \pi} \int_{0}^{\infty} \int_{0}^{\infty} \exp \left[-\frac{2 \gamma}{n \kappa_{n}} \frac{c(n, r)}{c(2, r)}\right. \\
& \times\left.\int_{0}^{2 \pi} \int_{0}^{\infty} \mathbf{1}\left\{H\left(\left(\begin{array}{c}
\cos \theta \\
\sin \theta
\end{array}\right), t\right) \cap\left[o, s\left(\begin{array}{c}
\cos \varphi \\
\sin \varphi
\end{array}\right), u\left(\begin{array}{c}
\cos \psi \\
\sin \psi
\end{array}\right)\right] \neq \varnothing\right\} t^{r-1} \mathrm{~d} t \mathrm{~d} \theta\right] \\
& \times u s|\sin (\varphi-\psi) u s|^{n-2} \mathrm{~d} u \mathrm{~d} s \mathrm{~d} \varphi \mathrm{d} \psi \\
&=b_{n, 2} \int_{0}^{2 \pi} \int_{0}^{2 \pi} 2 \int_{0}^{\infty} \int_{0}^{s} \exp \left[-\frac{2 \gamma}{n \kappa_{n}} \frac{c(n, r)}{c(2, r)}\right. \\
&\left.\times \int_{0}^{2 \pi} \int_{0}^{\infty} \mathbf{1}\left\{H\left(\left(\begin{array}{c}
\cos \theta \\
\sin \theta
\end{array}\right), t\right) \cap\left[o, s\left(\begin{array}{c}
\cos \varphi \\
\sin \varphi
\end{array}\right), u\left(\begin{array}{c}
\cos \psi \\
\sin \psi
\end{array}\right)\right] \neq \varnothing\right\} t^{r-1} \mathrm{~d} t \mathrm{~d} \theta\right] \\
& \times|\sin (\varphi-\psi)|^{n-2}(u s)^{n-1} \mathrm{~d} u \mathrm{~d} s \mathrm{~d} \varphi \mathrm{d} \psi,
\end{aligned}
$$

where the symmetry in $u$ and $s$ is used to justify the second equality. Hence, we derive

$$
\begin{gathered}
\mathbb{E}\left[V_{n}\left(Z_{0}\right)^{2}\right] \\
=2 b_{n, 2} \int_{0}^{2 \pi} \int_{0}^{2 \pi} \int_{0}^{\infty} \int_{0}^{s} \exp \left[-\frac{2 \gamma}{n \kappa_{n}} \frac{c(n, r)}{c(2, r)}\right. \\
\times \int_{0}^{2 \pi} \int_{0}^{\infty} \mathbf{1}\left\{H\left(\left(\begin{array}{c}
\cos (\theta-\psi) \\
\sin (\theta-\psi)
\end{array}\right), t\right)\right. \\
\left.\left.\cap\left[o, s\left(\begin{array}{c}
\cos (\varphi-\psi) \\
\sin (\varphi-\psi)
\end{array}\right),\left(\begin{array}{c}
u \\
0
\end{array}\right)\right] \neq \varnothing\right\} t^{r-1} \mathrm{~d} t \mathrm{~d} \theta\right] \\
\times u^{n-1} s^{n-1}|\sin (\varphi-\psi)|^{n-2} \mathrm{~d} u \mathrm{~d} s \mathrm{~d} \varphi \mathrm{d} \psi
\end{gathered}
$$




$$
\begin{aligned}
=4 \pi b_{n, 2} & \int_{0}^{2 \pi} \int_{0}^{\infty} \int_{0}^{s} \exp \left[-\frac{2 \gamma}{n \kappa_{n}} \frac{c(n, r)}{c(2, r)}\right. \\
\times & \left.\times \int_{0}^{2 \pi} \int_{0}^{\infty} \mathbf{1}\left\{H\left(\left(\begin{array}{c}
\cos \theta \\
\sin \theta
\end{array}\right), t\right) \cap\left[o, s\left(\begin{array}{c}
\cos \varphi \\
\sin \varphi
\end{array}\right),\left(\begin{array}{l}
u \\
0
\end{array}\right)\right] \neq \varnothing\right\} t^{r-1} \mathrm{~d} t \mathrm{~d} \theta\right] \\
=8 \pi b_{n, 2} & \int_{0}^{\pi} \int_{0}^{\infty} \int_{0}^{s-1} \exp \left[-\frac{2 \gamma}{n \kappa_{n}} \frac{c(n, r)}{c(2, r)}\right. \\
& \left.\times \int_{0}^{2 \pi} \int_{0}^{\infty} \mathbf{1}\left\{H\left(\left(\begin{array}{c}
\cos \theta \\
\sin \theta
\end{array}\right), t\right) \cap\left[o, s\left(\begin{array}{c}
\cos \varphi \\
\sin \varphi
\end{array}\right),\left(\begin{array}{l}
u \\
0
\end{array}\right)\right] \neq \varnothing\right\} t^{r-1} \mathrm{~d} t \mathrm{~d} \theta\right] \\
& \times s^{n-1} u^{n-1}(\sin \varphi)^{n-2} \mathrm{~d} u \mathrm{~d} s \mathrm{~d} \varphi .
\end{aligned}
$$

For the last equation, we used the fact that

$$
\begin{aligned}
& \int_{\pi}^{2 \pi} \int_{0}^{\infty} \int_{0}^{s} \exp \left[-\frac{2 \gamma}{n \kappa_{n}} \frac{c(n, r)}{c(2, r)}\right. \\
& \left.\times \int_{0}^{2 \pi} \int_{0}^{\infty} \mathbf{1}\left\{H\left(\left(\begin{array}{c}
\cos \theta \\
\sin \theta
\end{array}\right), t\right) \cap\left[o, s\left(\begin{array}{c}
\cos \varphi \\
\sin \varphi
\end{array}\right),\left(\begin{array}{l}
u \\
0
\end{array}\right)\right] \neq \varnothing\right\} t^{r-1} \mathrm{~d} t \mathrm{~d} \theta\right] \\
& \quad \times u^{n-1} s^{n-1}|\sin \varphi|^{n-2} \mathrm{~d} u \mathrm{~d} s \mathrm{~d} \varphi \\
& =\int_{0}^{\pi} \int_{0}^{\infty} \int_{0}^{s} \exp \left[-\frac{2 \gamma}{n \kappa_{n}} \frac{c(n, r)}{c(2, r)}\right. \\
& \left.\times \int_{0}^{2 \pi} \int_{0}^{\infty} \mathbf{1}\left\{H\left(\left(\begin{array}{c}
\cos \theta \\
\sin \theta
\end{array}\right), t\right) \cap\left[o, s\left(\begin{array}{c}
\cos \varphi \\
\sin \varphi
\end{array}\right),\left(\begin{array}{l}
u \\
0
\end{array}\right)\right] \neq \varnothing\right\} t^{r-1} \mathrm{~d} t \mathrm{~d} \theta\right] \\
& \times u^{n-1} s^{n-1}(\sin \varphi)^{n-2} \mathrm{~d} u \mathrm{~d} s \mathrm{~d} \varphi,
\end{aligned}
$$

which follows from the invariance of the inner integral under reflection of $\varphi$ and $\theta$ with respect to the first axis (that is, invariance with respect to replacing $\varphi$ by $-\varphi$ and $\theta$ by $-\theta$ ). This completes the proof.

In the expression found in Lemma 3 the indicator function depends on $\theta, t, s, u$, and $\varphi$. Its support can be determined explicitly. This is used in the proof of the following lemma, where it is shown how the integration with respect to $t$ in Lemma 3 can be carried out.

Lemma 4. For $u, s \in(0, \infty), u \leq s$, and $\varphi \in(0, \pi)$,

$$
\begin{gathered}
\int_{0}^{2 \pi} \int_{0}^{\infty} \mathbf{1}\left\{H\left(\left(\begin{array}{c}
\cos \theta \\
\sin \theta
\end{array}\right), t\right) \cap\left[o, s\left(\begin{array}{c}
\cos \varphi \\
\sin \varphi
\end{array}\right),\left(\begin{array}{l}
u \\
0
\end{array}\right)\right] \neq \varnothing\right\} t^{r-1} \mathrm{~d} t \mathrm{~d} \theta \\
=\frac{s^{r} c(2, r)}{r} F_{r}\left(\frac{u}{s}, \varphi\right) .
\end{gathered}
$$

Proof. The value of the integral on the left-hand side of the asserted equation does not change if we choose $[-\pi / 2,3 \pi / 2]$ instead of $[0,2 \pi]$ as the integration domain. Thus, we have to determine the support of the indicator function under the integral on $[-\pi / 2,3 \pi / 2] \times[0, \infty)$. Let $\varphi \in(0, \pi)$ be fixed. Then the indicator function is 1 if and only if

$$
H\left(\left(\begin{array}{c}
\cos \theta \\
\sin \theta
\end{array}\right), t\right) \cap\left[o, s\left(\begin{array}{c}
\cos \varphi \\
\sin \varphi
\end{array}\right)\right] \neq \varnothing \quad \text { or } \quad H\left(\left(\begin{array}{c}
\cos \theta \\
\sin \theta
\end{array}\right), t\right) \cap\left[o,\left(\begin{array}{l}
u \\
0
\end{array}\right)\right] \neq \varnothing
$$




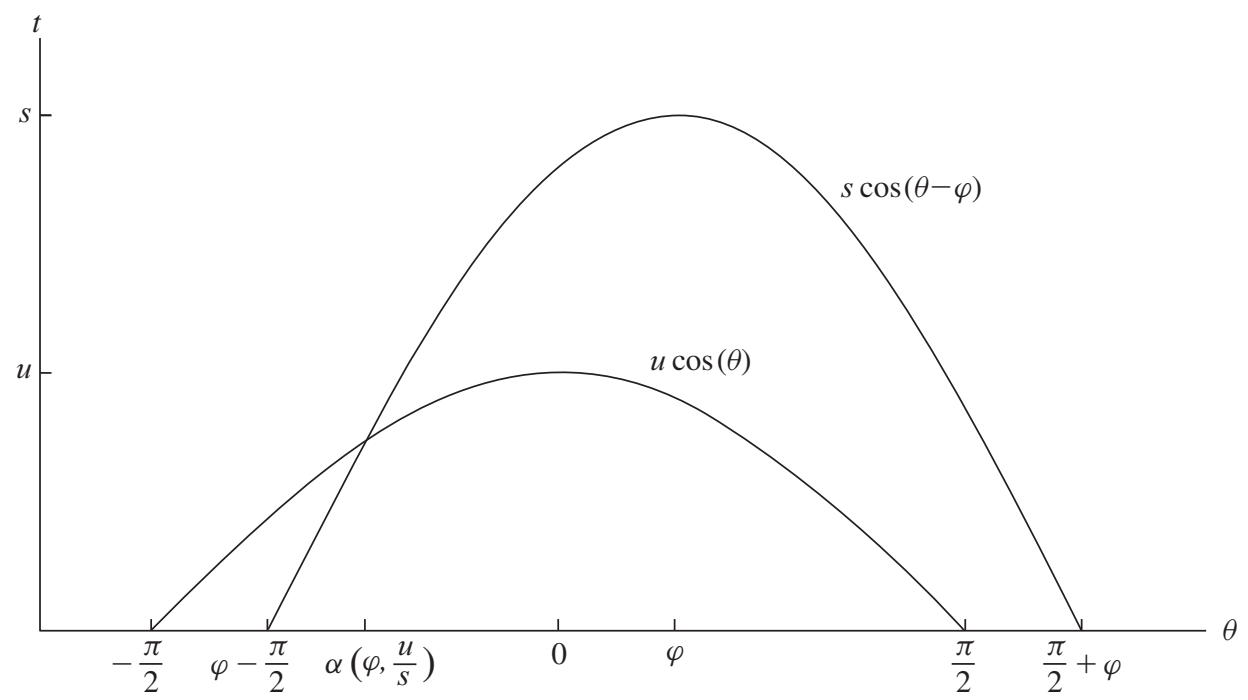

FIGURE 3: Support of the indicator function for $\varphi=\pi / 7$.

which is satisfied if and only if

$$
t \in\left[0, u(\cos \theta)_{+}\right] \cup\left[0, s(\cos (\theta-\varphi))_{+}\right] .
$$

If $\theta \in[-\pi / 2, \varphi-\pi / 2]$, this is equivalent to $t \in[0, u \cos \theta]$. If $\theta \in[\varphi-\pi / 2, \pi / 2]$, this is equivalent to $t \in[0, \max \{u \cos \theta, s \cos (\theta-\varphi)\}]$. If $\theta \in[\pi / 2, \varphi+\pi / 2]$, this is equivalent to $t \in[0, s \cos (\theta-\varphi)]$. For all other choices of $\theta \in[-\pi / 2,3 \pi / 2]$, this is equivalent to $t=0$, and, hence, can be neglected for the integration. Since

$$
u \cos \theta=s \cos (\theta-\varphi) \Longleftrightarrow \theta=\arctan \left(\frac{u / s-\cos \varphi}{\sin \varphi}\right)=\alpha\left(\frac{u}{s}, \varphi\right),
$$

we conclude that (7) is satisfied if and only if

$$
(\theta, t) \in\left(\left[-\frac{\pi}{2}, \alpha\left(\frac{u}{s}, \varphi\right)\right) \times[0, u \cos \theta]\right) \cup\left(\left[\alpha\left(\frac{u}{s}, \varphi\right), \varphi+\frac{\pi}{2}\right] \times[0, s \cos (\theta-\varphi)]\right),
$$

as illustrated in Figure 3. Now the integral can be easily computed.

\section{Variance inequalities}

In the next theorem, inequalities for $\operatorname{var}\left[V_{n}\left(Z_{0}\right)\right]$ are provided. In these inequalities two auxiliary quantities, $D(n, r)$ and $E(n, r)$, to be defined below, are involved. In Lemma 5 we establish crucial bounds for $E(n, r)$.

For $n \in \mathbb{N}$ with $n \geq 2$ and $r>0$, we define

$$
D(n, r):=\frac{n \kappa_{n}^{2}}{r} \Gamma\left(\frac{2 n}{r}+1\right)\left(\frac{n \kappa_{n} r}{4 \gamma c(n, r)}\right)^{2 n / r}
$$

and

$$
E(n, r):=\frac{n}{c(2, n-2)} \int_{0}^{\pi}(\sin \varphi)^{n-2} \int_{0}^{1} t^{n-1}\left(1+t^{r}-F_{r}(t, \varphi)\right) \mathrm{d} t \mathrm{~d} \varphi
$$


Introducing for $v \in[-\pi / 2, \pi / 2]$ and $r \in(0, \infty)$ the function $M(v, r)$ by

$$
M(v, r):=\frac{1}{c(2, r)} \int_{v}^{\pi / 2}(\cos \theta)^{r} \mathrm{~d} \theta
$$

we obtain

$$
\begin{aligned}
E(n, r)= & \frac{n}{c(2, n-2)} \\
& \times \int_{0}^{\pi}(\sin \varphi)^{n-2} \int_{0}^{1} t^{n-1}\left[t^{r} M(\alpha(t, \varphi), r)+M(\varphi-\alpha(t, \varphi), r)\right] \mathrm{d} t \mathrm{~d} \varphi,
\end{aligned}
$$

where $-\pi / 2<\alpha(t, \varphi)<\pi / 2$ and $0<\varphi / 2 \leq \varphi-\alpha(t, \varphi) \leq \pi / 2$ for $\varphi \in(0, \pi)$.

Theorem 2. With these definitions, we have

$$
E(n, r) D(n, r) \leq \operatorname{var}\left[V_{n}\left(Z_{0}\right)\right] \leq E(n, r) D(n, r) 4^{(2 n / r)+1} .
$$

Proof. The mean value theorem and (6) yield

$$
\begin{aligned}
\int_{0}^{\pi} & \int_{0}^{1}\left(\frac{1}{F_{r}(t, \varphi)^{2 n / r}}-\frac{1}{\left(t^{r}+1\right)^{2 n / r}}\right) t^{n-1}(\sin \varphi)^{n-2} \mathrm{~d} t \mathrm{~d} \varphi \\
& \geq \frac{n}{r} \frac{1}{2^{2 n / r}} \int_{0}^{\pi} \int_{0}^{1}\left(t^{r}+1-F_{r}(t, \varphi)\right) t^{n-1}(\sin \varphi)^{n-2} \mathrm{~d} t \mathrm{~d} \varphi
\end{aligned}
$$

hence, we deduce from Theorem 1 that

$$
\operatorname{var}\left[V_{n}\left(Z_{0}\right)\right] \geq E(n, r) \frac{c(2, n-2)}{r 2^{2 n / r}} \frac{8 \pi b_{n, 2}}{r} \Gamma\left(\frac{2 n}{r}\right)\left(\frac{n \kappa_{n} r}{2 \gamma c(n, r)}\right)^{2 n / r}=E(n, r) D(n, r) .
$$

Again, by the mean value theorem and (6), we have

$$
\begin{aligned}
& \int_{0}^{\pi} \int_{0}^{1}\left(\frac{1}{F_{r}(t, \varphi)^{2 n / r}}-\frac{1}{\left(t^{r}+1\right)^{2 n / r}}\right) t^{n-1}(\sin \varphi)^{n-2} \mathrm{~d} t \mathrm{~d} \varphi \\
& \quad \leq 4 \frac{n}{r} 2^{2 n / r} \int_{0}^{\pi} \int_{0}^{1}\left(t^{r}+1-F_{r}(t, \varphi)\right) t^{n-1}(\sin \varphi)^{n-2} \mathrm{~d} t \mathrm{~d} \varphi .
\end{aligned}
$$

Now the assertion follows in a similar way as for the lower bound.

The next lemma provides upper and lower bounds for the auxiliary quantity $E(n, r)$. In the sequel, these bounds will then be combined with Theorem 2 to obtain further consequences stated in Corollaries 1 and 2 and in Theorem 3.

Lemma 5. (a) For all $r \in(0, \infty)$, we have

$$
c_{r} \leq E(n, r) \leq \frac{3}{2}
$$

with a constant $c_{r}>0$ which depends on $r$ but not on $n$. 
(b) For all $r \in(0, \infty)$ and $n \geq 3$, we have

$$
\begin{aligned}
& c \frac{(1+r / n)^{-1 / 2}}{\sqrt{r+1}} 2^{n / 2}\left(1+\frac{r}{2 n}\right)^{-n / 2}\left(1+\frac{n}{n+r}\right)^{-(n+r) / 2} \\
& \quad \leq E(n, r) \\
& \quad \leq C \frac{(1+r / n)}{\sqrt{r+1}} 2^{n / 2}\left(1+\frac{r}{2 n}\right)^{-n / 2}\left(1+\frac{n}{n+r}\right)^{-(n+r) / 2}
\end{aligned}
$$

with constants $c, C>0$ which are independent of $r$ and $n$.

(c) For all $r \in(0, \infty)$, we have

$$
c \frac{1}{(r+1)^{2}} \leq E(2, r) \leq \frac{1}{\sqrt{r+1}}
$$

with a constant $c>0$ which is independent of $r$.

Proof. (a) Let $r \in(0, \infty)$ be fixed. By (6) and Remark 3(b), we obtain

$$
E(n, r) \leq \frac{n}{c(2, n-2)} \int_{0}^{\pi}(\sin \varphi)^{n-2} \int_{0}^{1} t^{n-1} \frac{3}{2} \mathrm{~d} t \mathrm{~d} \varphi=\frac{3}{2} .
$$

Next we bound $E(n, r)$ from below. For this, we start from (8), use the facts that $M$ is nonnegative and decreasing with respect to its first argument, and that $\alpha(t, \varphi) \leq \varphi / 2 \leq \pi / 4$ for $\varphi \in(0, \pi / 2)$ by Remark 3(a), and then apply Remark 3(b). This leads to

$$
\begin{aligned}
E(n, r) & \geq \frac{n}{c(2, n-2)} \int_{0}^{\pi / 2}(\sin \varphi)^{n-2} \int_{0}^{1} t^{n+r-1} M(\alpha(t, \varphi), r) \mathrm{d} t \mathrm{~d} \varphi \\
& \geq \frac{n}{c(2, n-2)} \int_{0}^{\pi / 2}(\sin \varphi)^{n-2} \mathrm{~d} \varphi \frac{M(\pi / 4, r)}{n+r} \\
& \geq(2(1+r))^{-1} M\left(\frac{\pi}{4}, r\right) \\
& =c_{r} \\
& >0 .
\end{aligned}
$$

(b) Note that

$$
E(n, r)=\frac{n}{c(2, n-2) c(2, r)} \int_{0}^{\pi}(\sin \varphi)^{n-2} \int_{0}^{1} t^{n-1} h_{\varphi}(t) \mathrm{d} t \mathrm{~d} \varphi,
$$

where $h_{\varphi}:[0,1] \rightarrow \mathbb{R}$ for $\varphi \in(0, \pi)$ is defined by

$$
h_{\varphi}(t):=c(2, r)\left(1+t^{r}-F_{r}(t, \varphi)\right), \quad t \in[0,1] .
$$

Observe that $h_{\varphi}$ depends on $r$, although this dependence is not made explicit by our notation (since $r>0$ is arbitrary but fixed in this part of the proof). Recall that $-\pi / 2<\alpha(t, \varphi)<\pi / 2$ and $0 \leq \varphi-\alpha(t, \varphi) \leq \pi / 2$, and note that

$$
h_{\varphi}(t)=t^{r} \int_{0}^{(\pi / 2)-\alpha(t, \varphi)}(\sin \theta)^{r} \mathrm{~d} \theta+\int_{0}^{(\pi / 2)-(\varphi-\alpha(t, \varphi))}(\sin \theta)^{r} \mathrm{~d} \theta .
$$


The function $h_{\varphi}$ is strictly increasing on $[0,1]$, since

$$
\begin{aligned}
h_{\varphi}^{\prime}(t) & =r t^{r-1} \int_{0}^{(\pi / 2)-\alpha(t, \varphi)}(\sin \theta)^{r} \mathrm{~d} \theta+\alpha_{t}(t, \varphi)\left((\cos (\alpha(t, \varphi)-\varphi))^{r}-t^{r}(\cos (\alpha(t, \varphi)))^{r}\right) \\
& =r t^{r-1} \int_{0}^{(\pi / 2)-\alpha(t, \varphi)}(\sin \theta)^{r} \mathrm{~d} \theta \\
& >0,
\end{aligned}
$$

where $0<\pi / 2-\alpha(t, \varphi)<\pi$. Here $\alpha_{t}$ denotes the partial derivative of $\alpha(t, \varphi)$ with respect to the first argument $t$. For the last equality, we used the fact that $\cos (\varphi)+\tan (\alpha(t, \varphi)) \sin \varphi=t$, by the definition of $\alpha(t, \varphi)$, and, therefore,

$$
\cos (\alpha(t, \varphi)-\varphi)=\cos (\alpha(t, \varphi)) \cos (\varphi)+\sin (\alpha(t, \varphi)) \sin \varphi=t \cos (\alpha(t, \varphi)) .
$$

Since $\alpha(1, \varphi)=\varphi / 2$, we obtain, for $t \in[0,1]$,

$$
0=h_{\varphi}(0) \leq h_{\varphi}(t) \leq h_{\varphi}(1)=2 \int_{0}^{(\pi-\varphi) / 2}(\sin \theta)^{r} \mathrm{~d} \theta,
$$

which implies that

$$
E(n, r) \leq \frac{2}{c(2, n-2) c(2, r)} \int_{0}^{\pi}(\sin \varphi)^{n-2} \int_{0}^{(\pi-\varphi) / 2}(\sin \theta)^{r} \mathrm{~d} \theta \mathrm{d} \varphi .
$$

Reproducing the argument at the bottom of page 925 of [1], for $\varphi \in(0, \pi)$, we obtain

$$
\begin{aligned}
\int_{0}^{(\pi-\varphi) / 2}(\sin \theta)^{r} \mathrm{~d} \theta & \leq \int_{0}^{(\pi-\varphi) / 2} \frac{\cos \theta}{\cos ((\pi-\varphi) / 2)}(\sin \theta)^{r} \mathrm{~d} \theta \\
& =\frac{(\sin ((\pi-\varphi) / 2))^{r+1}}{(r+1) \sin (\varphi / 2)} \\
& =\frac{(\cos (\varphi / 2))^{r+1}}{(r+1) \sin (\varphi / 2)}
\end{aligned}
$$

From (9) and (10), we deduce for $n \geq 3$ that

$$
\begin{aligned}
E(n, r) & \leq \frac{2}{(r+1) c(2, n-2) c(2, r)} \int_{0}^{\pi}(\sin \varphi)^{n-2} \frac{(\cos (\varphi / 2))^{r+1}}{\sin (\varphi / 2)} \mathrm{d} \varphi \\
& \leq \frac{2^{n}}{(r+1) c(2, n-2) c(2, r)} \int_{0}^{\pi / 2}(\sin \psi)^{n-3}(\cos \psi)^{n+r-1} \mathrm{~d} \psi \\
& =\frac{2^{n-1} \Gamma((n-2) / 2) \Gamma((n+r) / 2)}{(r+1) c(2, n-2) c(2, r) \Gamma(n+r / 2-1)} \\
& =\frac{1+(r+2) /(2(n-2))}{(r+1) \pi} 2^{n} \frac{\Gamma(n / 2)^{2} \Gamma((n+r) / 2) \Gamma(r / 2+1)}{\Gamma((n-1) / 2) \Gamma((2 n+r) / 2) \Gamma((r+1) / 2)} .
\end{aligned}
$$

Then (2) implies the inequality

$$
E(n, r) \leq C \frac{1+r / n}{\sqrt{r+1}} 2^{n / 2}\left(1+\frac{n}{n+r}\right)^{-(n+r) / 2}\left(1+\frac{r}{2 n}\right)^{-n / 2} .
$$


To derive a lower bound for $E(n, r)$ for all $n \geq 2$, we start from (8) to obtain

$$
E(n, r) \geq \frac{n}{c(2, n-2)} \int_{0}^{\pi}(\sin \varphi)^{n-2} \int_{0}^{1} t^{n+r-1} M(\alpha(t, \varphi), r) \mathrm{d} t \mathrm{~d} \varphi,
$$

since $M$ is nonnegative. For $\varphi \in(0, \pi)$, we have $\alpha(t, \varphi) \leq \varphi / 2$ and $\varphi / 2 \in(0, \pi / 2)$; hence, $M(\varphi / 2, r) \leq M(\alpha(t, \varphi), r)$. Thus, we obtain

$$
\begin{aligned}
M(\alpha(t, \varphi), r) & \geq M\left(\frac{\varphi}{2}, r\right) \\
& =\frac{1}{c(2, r)} \int_{\varphi / 2}^{\pi / 2}(\cos \theta)^{r} \mathrm{~d} \theta \\
& \geq \frac{1}{c(2, r)} \int_{\varphi / 2}^{\pi / 2}(\cos \theta)^{r} \sin \theta \mathrm{d} \theta \\
& =\frac{1}{c(2, r)} \frac{1}{r+1}\left(\cos \frac{\varphi}{2}\right)^{r+1} .
\end{aligned}
$$

This implies that

$$
\begin{aligned}
E(n, r) & \geq \frac{n}{n+r} \frac{1}{r+1} \frac{1}{c(2, n-2) c(2, r)} \int_{0}^{\pi}(\sin \varphi)^{n-2}\left(\cos \frac{\varphi}{2}\right)^{r+1} \mathrm{~d} \varphi \\
& \geq c \frac{n}{n+r} \sqrt{\frac{n}{r+1}} 2^{n-1} \int_{0}^{\pi / 2}(\sin \psi)^{n-2}(\cos \psi)^{n+r-1} \mathrm{~d} \psi \\
& =c\left(1+\frac{r}{n}\right)^{-1} \sqrt{\frac{n}{r+1}} 2^{n-2} \frac{\Gamma((n-1) / 2) \Gamma((n+r) / 2)}{\Gamma((2 n+r-1) / 2)}
\end{aligned}
$$

The approximation (2) of the gamma function then yields

$$
\begin{aligned}
E(n, r) & \geq c \frac{(1+r / n)^{-1}}{\sqrt{r+1}} 2^{n / 2}\left(1+\frac{r+1}{2(n-1)}\right)^{-(n-1) / 2}\left(1+\frac{n-1}{n+r}\right)^{-(n+r) / 2} \\
& \geq c \frac{(1+r / n)^{-1 / 2}}{\sqrt{r+1}} 2^{n / 2}\left(1+\frac{r}{2 n}\right)^{-n / 2}\left(1+\frac{n}{n+r}\right)^{-(n+r) / 2},
\end{aligned}
$$

which gives the lower bound.

(c) Since the proof of the lower bound in (b) works also for $n=2$, we immediately obtain the lower bound.

To derive the upper bound for $n=2$, we first use (9) to obtain

$$
\begin{aligned}
E(2, r) & \leq \frac{2}{\pi c(2, r)} \int_{0}^{\pi} \int_{0}^{(\pi-\varphi) / 2}(\sin \theta)^{r} \mathrm{~d} \theta \mathrm{d} \varphi \\
& =\frac{4}{\pi c(2, r)} \int_{0}^{\pi / 2}\left(\frac{\pi}{2}-\theta\right)(\sin \theta)^{r} \mathrm{~d} \theta \\
& =\frac{2}{c(2, r)} \int_{0}^{\pi / 2} \frac{2}{\pi} \vartheta(\cos \vartheta)^{r} \mathrm{~d} \vartheta .
\end{aligned}
$$


Since $2 \vartheta / \pi \leq \sin \vartheta$ for $\vartheta \in[0, \pi / 2]$, we further deduce that

$$
E(2, r) \leq \frac{2}{c(2, r)} \int_{0}^{\pi / 2} \sin \vartheta(\cos \vartheta)^{r} \mathrm{~d} \vartheta=\frac{2}{c(2, r)} \frac{1}{r+1} .
$$

Using (2), we find that $c(2, r) \geq 2 / \sqrt{r+1}$, which yields $E(2, r) \leq 1 / \sqrt{r+1}$.

In the following corollary, the bounds in (11) are obtained from Proposition 1 by applying (2); see (15), (16), and (17). For the bounds in (12), we first use Theorem 2. Then Lemma 5(a) implies that the factor $E(n, r)$ is bounded from above and below by constants depending only on $r$, whereas the factor $D(n, r)$ is equal to the upper bound from Proposition 1 for $k=2$ multiplied with $(n / r)(1 / 2)^{(2 n) / r}$, which has already been considered to obtain (11).

Corollary 1. For $k \in \mathbb{N}$ and fixed $r \in(0, \infty)$, there are constants $c_{r}, C_{r}>0$, depending on $r$ and $k$ but not on $n$ or $\gamma$, such that

$$
c_{r}\left(A(r) \frac{n}{\gamma}\left(1+\frac{r}{n}\right)^{n / 2}\right)^{k n / r} \leq \mathbb{E}\left[V_{n}\left(Z_{0}\right)^{k}\right] \leq C_{r} n^{(1-k) / 2}\left(A(r) \frac{k n}{\gamma}\left(1+\frac{r}{n}\right)^{n / 2}\right)^{k n / r}
$$

and there are constants $c_{r}, C_{r}>0$, depending on $r$ but not on $n$ or $\gamma$, such that

$$
c_{r} \sqrt{n}\left(A(r) \frac{n}{\gamma}\left(1+\frac{r}{n}\right)^{n / 2}\right)^{2 n / r} \leq \operatorname{var}\left[V_{n}\left(Z_{0}\right)\right] \leq C_{r} \sqrt{n}\left(A(r) \frac{4 n}{\gamma}\left(1+\frac{r}{n}\right)^{n / 2}\right)^{2 n / r},
$$

where

$$
A(r):=\frac{\pi^{(r+1) / 2}}{\mathrm{e} \Gamma((r+1) / 2)} .
$$

For constant intensity $\gamma$, we infer from Corollary 1 that $\mathbb{E}\left[V_{n}\left(Z_{0}\right)^{k}\right]$ and $\operatorname{var}\left[V_{n}\left(Z_{0}\right)\right]$ go to $\infty$ for fixed distance exponent $r$ and $n \rightarrow \infty$. Therefore, we now choose the intensity $\gamma$ as a function of $n$ and $r$ such that $\mathbb{E}\left[V_{n}\left(Z_{0}\right)\right]$ is equal to a positive constant $\lambda^{-1}$. By Proposition 1 we have

$$
\mathbb{E}\left[V_{n}\left(Z_{0}\right)\right]=\Gamma\left(\frac{n}{r}+1\right) \kappa_{n}\left(\frac{n \kappa_{n} r}{2 \gamma c(n, r)}\right)^{n / r} .
$$

Therefore, if we define

$$
\widehat{\gamma}(r, n):=\frac{n \kappa_{n} r}{2 c(n, r)}\left(\lambda \Gamma\left(\frac{n}{r}+1\right) \kappa_{n}\right)^{r / n}
$$

with $\lambda>0$ then

$$
\mathbb{E}\left[V_{n}\left(Z_{0}\right)\right]=\frac{1}{\lambda}
$$

If we substitute $\widehat{\gamma}(r, n)$ into the lower estimate from Theorem 2, keeping in mind that, by Lemma 5(a), the factor $E(n, r)$ is bounded from below by a constant depending only on $r$, and if we apply (2), then it follows that there is a constant $c_{r}>0$ depending on $r$ but not on $n$ or $\lambda$ such that

$$
\operatorname{var}\left[V_{n}\left(Z_{0}\right)\right] \geq c_{r} \frac{1}{r} \frac{n}{\lambda^{2}} \frac{\Gamma(2 n / r+1)}{\Gamma(n / r+1)^{2}} 2^{-2 n / r} \geq c_{r} \lambda^{-2} \sqrt{n} \rightarrow \infty \quad \text { as } n \rightarrow \infty .
$$

The preceding analysis suggests that in order to arrive at a limiting behaviour comparable to the case of a Poisson-Voronoi tessellation, i.e. with the variance converging to 0 as $n \rightarrow \infty$, 
we cannot choose the distance parameter $r$ fixed but have to adjust it to the dimension. In fact, in the following theorem we consider the case where $r$ is proportional to $n$, which is the natural choice in view of the estimates that have been obtained.

Corollary 2. Let $r=$ an with fixed $a \in(0, \infty)$ and $k \in \mathbb{N}$. Then there are constants $c, C>0$, depending on $a$ and $k$ but not on $n$ or $\gamma$, such that

$$
c \gamma^{-k / a} n^{k / a-k / 2}\left(\frac{B(a)}{n}\right)^{k n / 2} \leq \mathbb{E}\left[V_{n}\left(Z_{0}\right)^{k}\right] \leq C \gamma^{-k / a} n^{k / a-k / 2}\left(\frac{B(a)}{n}\right)^{k n / 2},
$$

and there are constants $c, C>0$, depending on a but not on $n$ or $\gamma$, such that

$$
\begin{aligned}
& c\left[\frac{4(a+1)^{a+1}}{(a+2)^{a+2}}\right]^{n / 2} \gamma^{-2 / a} n^{2 / a-3 / 2}\left(\frac{B(a)}{n}\right)^{n} \\
& \quad \leq \operatorname{var}\left[V_{n}\left(Z_{0}\right)\right] \\
& \quad \leq C\left[\frac{4(a+1)^{a+1}}{(a+2)^{a+2}}\right]^{n / 2} \gamma^{-2 / a} n^{2 / a-3 / 2}\left(\frac{B(a)}{n}\right)^{n},
\end{aligned}
$$

where

$$
B(a):=\frac{2 \pi \mathrm{e}(a+1)^{(a+1) / a}}{a} .
$$

Hence, if the intensity $\gamma$ is constant and $r=$ an then $\mathbb{E}\left[V_{n}\left(Z_{0}\right)^{k}\right]$ and $\operatorname{var}\left[V_{n}\left(Z_{0}\right)\right]$ converge to 0 as $n \rightarrow \infty$.

Proof. The inequalities in (13) follow from Proposition 1 by means of (2); see (15).

For the inequalities in (14), we first use Theorem 2. There is nothing to prove for $n=2$. Hence, we assume that $n \geq 3$. Then $E(n, a n)$ is bounded by means of Lemma 5(b), whereas the factor $D(n, a n)$ is equal to the upper bound from Proposition 1 for $k=2$ multiplied by $(1 / a)(1 / 2)^{2 / a}$, which has already been considered to obtain (13).

Remark 4. Note that $4(a+1)^{a+1} /(a+2)^{a+2}<1$ for $a>0$.

Now we again choose $r=a n$, with fixed $a>0$, and determine the intensity $\gamma$ in such a way that the expected volume of the zero cell is equal to a positive constant. This is possible for a special intensity function depending on $a$, the dimension $n$, and a positive constant $\lambda$ that can be prescribed arbitrarily. In the following theorem, we describe the asymptotic behaviour of the volume of the zero cell in such a setting.

Theorem 3. Let $r=$ an, with constant $a \in(0, \infty)$, and let the intensity be chosen as

$$
\widehat{\gamma}(a, n)=\frac{a n^{2} \kappa_{n}}{2 c(n, a n)}\left(\lambda \Gamma\left(\frac{1}{a}+1\right) \kappa_{n}\right)^{a}
$$

with $\lambda>0$. Then the following statements hold.

(a) For $k \in \mathbb{N}$,

$$
\frac{1}{\lambda^{k}} \leq \mathbb{E}\left[V_{n}\left(Z_{0}\right)^{k}\right] \leq \frac{\Gamma(k / a+1)}{\lambda^{k} \Gamma(1 / a+1)^{k}} ;
$$

in particular,

$$
\mathbb{E}\left[V_{n}\left(Z_{0}\right)\right]=\frac{1}{\lambda}
$$


(b) There are constants $c, C>0$, depending on a but not on $n$ or $\lambda$, such that

$$
c \frac{1}{\lambda^{2}} \frac{1}{\sqrt{n}}\left[\frac{4(a+1)^{a+1}}{(a+2)^{a+2}}\right]^{n / 2} \leq \operatorname{var}\left[V_{n}\left(Z_{0}\right)\right] \leq C \frac{1}{\lambda^{2}} \frac{1}{\sqrt{n}}\left[\frac{4(a+1)^{a+1}}{(a+2)^{a+2}}\right]^{n / 2} ;
$$

in particular,

$$
\lim _{n \rightarrow \infty} \operatorname{var}\left[V_{n}\left(Z_{0}\right)\right]=0 .
$$

Proof. The inequalities in (a) follow from Proposition 1 and the special choice of the intensity as $\widehat{\gamma}(a, n)$. In (b) there is nothing to prove for $n=2$. For $n \geq 3$, the inequalities follow from Theorem 2, since $D(n, a n)$ is proportional to $\lambda^{-2}$ for the intensity chosen as $\widehat{\gamma}(a, n)$ and by using the bounds for $E(n, a n)$ provided by Lemma 5(b).

Remark 5. For $a=1$, we have $\widehat{\gamma}(1, n)=n \kappa_{n} 2^{n-1} \lambda$ and Theorem 3 recovers the sharp bounds obtained by Alishahi and Sharifitabar [1].

\section{Appendix A. Auxiliary inequalities}

The subsequent inequalities follow from approximation (2) of the gamma function (see [2, p. 24] or [26, Equation (12.33)]). As before, let $r \in(0, \infty)$. Then

$$
\begin{aligned}
& \frac{\sqrt{\pi} r}{\Gamma((r+1) / 2)(2 \mathrm{e})^{r / 2}}\left(\frac{n+r}{n}\right)^{(n-1) / 2}(n+r)^{r / 2} \mathrm{e}^{-1 /(6 n)} \\
& \quad \leq \frac{n \kappa_{n} r}{2 c(n, r)} \\
& \quad \leq \frac{\sqrt{\pi} r}{\Gamma((r+1) / 2)(2 \mathrm{e})^{r / 2}}\left(\frac{n+r}{n}\right)^{(n-1) / 2}(n+r)^{r / 2} \mathrm{e}^{1 /(6(n+r))} .
\end{aligned}
$$

Furthermore, for $k \in \mathbb{N}$, we have

$$
\begin{aligned}
\sqrt{\frac{2 \pi}{r \mathrm{e}^{2}}} & \left(\frac{\mathrm{e}^{2}}{\pi}\right)^{k / 2} \frac{\sqrt{k n+r}}{(n+2)^{k / 2}}\left(\frac{\sqrt{2 \mathrm{e} \pi}}{(r \mathrm{e} / k)^{1 / r}}\right)^{n k} \mathrm{e}^{-k /(6(n+2))}\left(\left(n+\frac{r}{k}\right)^{1 / r}(n+2)^{-1 / 2}\right)^{n k} \\
& \leq \Gamma\left(\frac{k n}{r}+1\right) \kappa_{n}^{k} \\
& \leq \sqrt{\frac{2 \pi}{r \mathrm{e}^{2}}}\left(\frac{\mathrm{e}^{2}}{\pi}\right)^{k / 2} \frac{\sqrt{k n+r}}{(n+2)^{k / 2}}\left(\frac{\sqrt{2 \mathrm{e} \pi}}{(r \mathrm{e} / k)^{1 / r}}\right)^{n k} \mathrm{e}^{r /(12(k n+r))}\left(\left(n+\frac{r}{k}\right)^{1 / r}(n+2)^{-1 / 2}\right)^{n k}
\end{aligned}
$$

and

$$
\begin{aligned}
& \Gamma\left(\frac{n}{r}+1\right)^{k} \kappa_{n}^{k} \\
& \quad \geq\left(\frac{2}{r}\right)^{k / 2}\left(\frac{n+r}{n+2}\right)^{k / 2}\left(\frac{\sqrt{2 \mathrm{e} \pi}}{(r \mathrm{e})^{1 / r}}\right)^{n k} \mathrm{e}^{-k /(6(n+2))}\left((n+r)^{1 / r}(n+2)^{-1 / 2}\right)^{n k} .
\end{aligned}
$$

\section{Acknowledgements}

The authors would like to thank an anonymous referee and an editor for their useful comments which helped to improve the manuscript, and Walter Mickel (Karlsruhe Institute of Technology, Department of Mathematics) for his advice concerning the numerical calculations. 
The authors acknowledge support by the German research foundation (DFG) through the research group 'Geometry and Physics of Spatial Random Systems' under grants HU1874/2-1 and HU1847/3-1.

\section{References}

[1] Alishahi, K. and Sharifitabar, M. (2008). Volume degeneracy of the typical cell and the chord length distribution for Poisson-Voronoi tessellations in high dimensions. Adv. Appl. Prob. 40, 919-938.

[2] Artin, E. (1964). The Gamma Function. Holt, Rinehart and Winston, New York.

[3] Bárány, I. (2008). Random points and lattice points in convex bodies. Bull. Amer. Math. Soc. (N.S.) 45, 339-365.

[4] Calka, P. (2010). Tessellations. In New Perspectives in Stochastic Geometry, eds W. S. Kendall and I. Molchanov, Oxford University Press, pp. 145-169.

[5] Federer, H. (1969). Geometric Measure Theory. Springer, New York.

[6] Hilhorst, H. J. and Calka, P. (2008). Random line tessellations of the plane: statistical properties of manysided cells. J. Statist. Phys. 132, 627-647.

[7] Hug, D. (2007). Random mosaics. In Stochastic Geometry (Lecture Notes Math. 1892), ed. W. Weil, Springer, Berlin, pp. 247-266.

[8] Hug, D. And Schneider, R. (2007). Asymptotic shapes of large cells in random tessellations. Geom. Funct. Anal. 17, 156-191.

[9] Hug, D. And Schneider, R. (2010). Large faces in Poisson hyperplane mosaics. Ann. Prob. 38, $1320-1344$.

[10] Hug, D., Reitzner, M. And Schneider, R. (2004). The limit shape of the zero cell in a stationary Poisson hyperplane tessellation. Ann. Prob. 32, 1140-1167.

[11] Mecke, J. (1998). Inequalities for mixed stationary Poisson hyperplane tessellations. Adv. Appl. Prob. 30, 921-928.

[12] Mecke, J. (1999). On the relationship between the 0-cell and the typical cell of a stationary random tessellation. Pattern Recogn. 32, 1645-1648.

[13] MøLler, J. (1994). Lectures on Random Voronǒ Tessellations (Lecture Notes Statist. 87). Springer, New York.

[14] Muche, L. (2010). Contact and chord length distribution functions of the Poisson-Voronoi tessellation in high dimensions. Adv. Appl. Prob. 42, 48-68.

[15] Muche, L. and Ballani, F. (2011). The second volume moment of the typical cell and higher moments of edge lengths of the spatial Poisson-Voronoi tessellation. Monatsh. Math. 163, 71-80.

[16] Müller, C. (1998). Analysis of Spherical Symmetries in Euclidean Spaces (Appl. Math. Sci. 129). Springer, New York.

[17] Newman, C. M. And RinotT, Y. (1985). Nearest neighbors and Voronoř volumes in high-dimensional point processes with various distance functions. Adv. Appl. Prob. 17, 794-809.

[18] Newman, C. M., Rinott, Y. And Tversky, A. (1983). Nearest neighbors and Voronoǐ regions in certain point processes. Adv. Appl. Prob. 15, 726-751.

[19] OKabe, A., Boots, B., Sugihara, K. And Chiu, S. N. (2000). Spatial Tessellations: Concepts and Applications of Voronoi Diagrams, 2nd edn. John Wiley, Chichester.

[20] Reitzner, M. (2010). Random Polytopes. In New Perspectives in Stochastic Geometry, eds W. S. Kendall and I. Molchanov, Oxford University Press, pp. 45-76.

[21] Schneider, R. (2003). Nonstationary Poisson hyperplanes and their induced tessellations. Adv. Appl. Prob. 35, $139-158$.

[22] Schneider, R. (2008). Recent results on random polytopes. Boll. Unione. Mat. Ital. 1, 17-39.

[23] Schneider, R. And WeIL, W. (2008). Stochastic and Integral Geometry. Springer, Berlin.

[24] Spodarev, E. (ed.) (2013). Stochastic Geometry, Spatial Statistics and Random Fields (Lecture Notes Math. 2068). Springer, Berlin.

[25] Stoyan, D., Kendall, W. S. And Mecke, J. (1995). Stochastic Geometry and Its Applications. 2nd edn. John Wiley, Chichester.

[26] Whittaker, E. T. and Watson, G. N. (1996). A Course of Modern Analysis. Reprint of the 4th (1927) edn. Cambridge University Press.

[27] YAO, Y.-C. (2010). On variances of partial volumes of the typical cell of a Poisson-Voronoi tessellation and large-dimensional volume degeneracy. Adv. Appl. Prob. 42, 359-370. 